\title{
图HAD
}

ISSN-L: 2530-5115

DOI: http://doi.org/10.22585/hospdomic.v4i3.107

\section{Recomendaciones para la atención kinésica respiratoria en el contexto de paciente sospechoso y confirmado para Covid-19 en las unidades de Hospitalización Domiciliaria}

Recommendations for Respiratory Physiotherapy in the Context of Suspicious and Confirmed Patient for Covid-19 in Hospital at Home

Francisco Freire Figueroa', Viviana Marín Navarro', Camilo Villarroel Sgorbini', Christian Poblete Figueroa', Evelyn Guzmán Llorens², Cesar Villagrán Azocar², Camila Monardes Cataldo' ${ }^{2}$ Daniela Alvear Soto ${ }^{3}$, David Figueroa Poblete ${ }^{4}$, Monserrat Viveros Vejar ${ }^{5}$, Danilo Méndez Yañez ${ }^{5}$, Rocío Diaz Oyarzo ${ }^{6}$, Felipe Cabezas Arane$\mathrm{da}^{7}$, Alexis Rojas Ojeda ${ }^{8}$, Lorena Guerrero Covarrubias9, Carlos Barraza Muñoz Nathaly Bacho Palacios ${ }^{10}$, Víctor Bascuñán Vivallo ${ }^{11}$. Eduardo Molina Morales ${ }^{12}$
1. Complejo Asistencial Dr. Sotero del Rio. Hospitalización Domiciliaria. Santiago de Chile.
2. Hospital San Juan de Dios. Hospitalización Domiciliaria. Santiago, Chile.
3. Hospital Sanatorio el Pino. Hospitalización Domiciliaria. Santiago, Chile.
4. Hospital base de Valdivia. Hospitalización Domiciliaria. Valdivia, Chile.
5. Hospital clínico Herminda Martin. Hospitalización Domiciliaria. Chillan, Chile.
6. Hospital Eduardo Schutz Schroeder. Hospitalización Domiciliaria. Puerto Montt, Chile.
7. Complejo Asistencial Dr. Víctor Ríos Ruiz Hospitalización Domiciliaria. Los Ángeles, Chile.
8. Hospital Dr. Ernesto Torres Galdames. Hospitalización Domiciliaria, lquique, Chile.
9. Hospital San Juan de Dios. Hospitalización Domiciliaria. La Serena, Chile.
10. Hospital del Salvador. Hospitalización Domiciliaria. Santiago, Chile.
11. Hospital Padre Hurtado Hospitalización Domiciliaria. Santiago, Chile.
12. Hospital de Quilpué, Hospitalización Domiciliaria. Quilpué, Chile.

Correspondencia/Correspondence

Francisco Freire Figueroa

f.freire.figueroa@gmail.com

Recibido/Received

20.05.2020

Aceptado/Accepted

25.05.2020
Conflicto de Intereses/Competing interest Se declara la inexistencia de todo tipo de conflicto de interés

Financiación/Fundings

No financiado

Agradecimientos/Acknowledgments

El presente trabajo cuenta con el respaldo de Sociedad Chilena de Kinesiología Respiratoria (SOCHIKIR) 
CÓMO CITAR ESTE TRABAJO | HOW TO CITE THIS PAPER

Freire-Figueroa F, Marín-Navarro V, Villarroel-Sgorbini C, Poblete-Figueroa C, Guzmán-Llorens E, Villagrán-Azocar $C$, et al. Recomendaciones para la atención kinésica respiratoria en el contexto de paciente sospechoso y confirmado para Covid-19 en las unidades de Hospitalización Domiciliaria. Hosp Domic. 2020;4(3): 133-52.

\section{RESUMEN}

\section{Propósito}

Ante la crisis sanitaria que vive el país y el mundo actualmente, se confeccionó este documento por la iniciativa autónoma de kinesiólogos de doce unidades de Hospitalización Domiciliaria (HD) de hospitales públicos de Chile, con el fin de unificar criterios en base a sus experiencias y la evidencia científica disponible para poder realizar una atención kinésica respiratoria segura en pacientes con sospecha o confirmados para COVID-19. Además, este documento tiene el objetivo de ser una guía para quienes tengan la necesidad de protocolizar y/o estandarizar su atención en HD, pudiendo ser adaptado según la necesidad y disponibilidad de recursos e infraestructura de cada unidad. Es importante señalar, que este documento puede variar según la evolución de esta pandemia y la actualización de la evidencia científica.

\section{Campo de aplicación}

Este documento está diseñado para ser aplicado en el domicilio de los pacientes adultos y pediátricos ingresados a las Unidades de Hospitalización Domiciliaria (UHD) con sospecha de infección por SARS-CoV-2 o con resultado positivo para COVID-19.

\section{ABSTRACT}

\section{Porpuse}

Given the health crisis that the country and the world are currently experiencing, this document was prepared by the autonomous initiative of kinesiologists from twelve Hospital at Home units of public hospitals in Chile, in order to unify criteria based on their experiences and the available scientific evidence in order to carry out safe respiratory physiotherapy care in patients with suspected or confirmed COVID-19. In addition, this document is intended to be a guide for those who need to protocolize and / or standardize their care at Hospital at home, and can be adapted according to the need and availability of resources and infrastructure of each unit. It is important to note that this document may vary depending on the evolution of this pandemic and the updating of scientific evidence.

\section{Scope}

This document is designed to be applied in the home of adult and pediatric patients admitted to Hospital at Home Units (UHD) with suspected SARS-CoV-2 infection or with a positive result for COVID-19 


\section{TERMINOLOGÍA}

- Coronavirus y SARS-CoV-2: Los coronavirus son virus de ARN de cadena positiva y sencilla, no segmentados. Pertenecen a la familia Coronaviridae y llevan su nombre por las protuberancias en forma de corona en la envoltura del virus. El SARS-CoV-2 Es un nuevo Coronavirus identificado en casos de neumonía grave que se iniciaron en China el año 2019. Responsable de la pandemia mundial declarada por la Organización Mundial de la Salud (OMS) en marzo de 2020. El periodo medio desde el inicio de los síntomas hasta la recuperación es de 2 semanas cuando la enfermedad ha sido leve y de 3-6 semanas cuando ha sido grave o crítica. El período medio de incubación es de 5 días y en el $98 \%$ de los casos los síntomas aparecen entre el día 2 y el 14 (1-4).

- COVID-19: Es la enfermedad infecciosa causada por el virus SARS-CoV-2. COVID-19 es su acrónimo en inglés (coronavirus disease 2019), también conocida como enfermedad por coronavirus $(5,6)$.

- Hospitalización Domiciliaria (HD): Se define como el traslado al domicilio del paciente, el personal, los servicios y la tecnología necesaria para su recuperación o tratamiento en igual cantidad y calidad que en el hospital, propiciando la participación del paciente y su familia en el proceso terapéutico $(3,7)$.

- Precauciones Estándar: Conjunto de medidas que tienen por objetivo prevenir la transmisión de la mayoría de los agentes microbianos durante la atención en salud, en particular la transmisión por las manos del personal y/o uso de equipos clínicos. Se aplican a la atención de todos los pacientes, independiente de si se trata o no de pacientes infecciosos, si se conoce o no su estado de portador del agente y/o si están sintomáticos o asintomáticos $(8,9)$.

- Equipo de protección personal (EPP): Conjunto de elementos de equipamiento, componentes de las precauciones estándar, destinados a proteger la piel y mucosas del operador en forma de barrera y evitar que se ponga en contacto con los agentes infecciosos $(5,9)$.

- Pacientes sintomáticos: Pacientes que presentan principalmente síntomas tales como temperatura corporal sobre $37,8^{\circ} \mathrm{C}$, tos seca o con esputo, mialgia, odinofagia, disnea y/o diarrea $(2,5)$.

- Pacientes asintomáticos: Pacientes positivos a la prueba de PCR para COVID-19 y que no presentan síntomas descritos anteriormente. Este tipo de pacientes mantienen la misma carga viral que un paciente sintomático por lo que los cuidados de prevención de los profesionales deben ser los mismos $(2,6)$.

- Kinesiología Respiratoria (KTR): Es la especialidad que poseen los kinesiólogos en el manejo de técnicas y procedimientos preventivos y/o terapéuticos en pacientes con patologías respiratorias, con el objetivo de restaurar la función pulmonar (10).

- En HD se incluye el uso de equipos de oxigenoterapia, aerosol terapia, bomba de aspiración de secreciones y manejo integral avanzado de la vía aérea (Traqueostomía). Además de equipamiento y personal capacitado para la utilización de ventilación mecánica no invasiva (VMNI), ventilación mecánica invasiva (VMI) y cánula nasal de alto flujo (CNAF) (7).

- Rehabilitación cardiorrespiratoria: Rehabilitación kinésica, que busca a través del ejercicio terapéutico correctamente indicado, optimizar el consumo de oxígeno (VO2) del paciente para finalmente mejorar la funcionalidad de este (11).

\section{MATERIALES}


Los materiales aquí expuestos deben ser los mínimos para trabajar en un ambiente domiciliario seguro con este tipo de pacientes. De todos modos, pueden ser remplazados según disponibilidad de cada HD.

- Bomba portátil de aspiración de secreciones.

- Oxímetro de pulso.

- Tensiómetro.

- Termómetro.

- Mascarilla de nebulización.

- Insumos para manejo de vía aérea artificial, principalmente TQT.

- Aerocámara.

- Equipos de oxigenoterapia.

- Nebulizador portátil.

- Baja lengua.

- Guantes de procedimiento.

- Guantes estériles.

- Mascarilla quirúrgica simple.

- Mascarilla N95 o superior.

- Pechera plástica impermeable, desechable de manga larga y/o traje de seguridad desechable.

- Protección ocular (antiparras) y/o escudo facial.

- Cofias o cubre cabellera desechable.

- Material de limpieza de equipos (alcohol 70\%).

- Contenedor de residuos.

\section{ACTIVIDADES GENERALES}

Se asume que el paciente cumple con los criterios de ingreso a kinesiología descrito por su unidad según recursos y estabilidad clínica.

\section{Recomendaciones generales}

Se busca dar cumplimiento a estas recomendaciones, considerando que pueden variar según la evidencia científica disponible, las características del paciente y el lugar de vivienda.

\section{Seguimiento telefónico}

- En pacientes confirmados con COVID-19 o con sospecha a la espera del resultado, con indicación médica de kinesiología respiratoria se privilegiará el seguimiento telefónico, pesquisando criterios de estabilidad clínica según ANEXO $N^{\circ} 1$, solo realizando visitas específicas frente a la alteración de estos criterios.

- El paciente y/o tutor debe contar con un teléfono que garantice la comunicación con el personal kinésico a diario.

- Reforzar en cada control telefónico respecto a signos y síntomas de alerta: fiebre mayor o igual a $37,8^{\circ} \mathrm{C}$ que no cede con paracetamol, dolor de garganta, dolores musculares, 
tos y dificultad para respirar (1-3). Además, se debe registrar cualquier otro síntoma que manifieste el paciente.

- En caso de que paciente no responda en reiteradas ocasiones, se coordinará su seguimiento, en conjunto con equipo de enfermería y/o área social.

\section{Atención en domicilio}

- Las visitas deben estar programadas y organizadas con antelación según necesidades del paciente, evitando así visitar más de una vez por día y privilegiando el profesional que sea más competente según su estado clínico.

- Revisar antecedentes en ficha clínica y realizar llamado telefónico mínimo una hora antes de la visita para solicitar y/o recordar que deben contar con jabón líquido y toalla de papel, además de ventilar la habitación, realizar aseo del baño y suspender la alimentación, esto último principalmente en paciente pediátricos; se recomienda también solicitar que sea la familia quien espere a los funcionarios con las puertas abiertas y así evitar el contacto con estructuras contaminadas.

- Se deben retirar relojes, aros, collares, pulseras y anillos previo a la atención clínica.

- El personal debe tener el cabello amarrado e idealmente afeitados.

- No se debe portar teléfono celular ni otros objetos susceptibles de contaminación, como por ejemplo credencial colgante.

- Se reducirá al mínimo el número de personas en la habitación del paciente, en lo posible solo el kinesiólogo/a y un asistente (TENS, Enfermero/a o familiar) en caso de ser necesario. Para este último caso ambos deberán llevar sus EPP correspondientes (Anexo º2)

- Las medidas de prevención, EPP y lavado clínico de manos en lo posible se deben realizar fuera del domicilio. Se debe descender del vehículo sólo con los insumos indispensables para la atención.

- En el domicilio, el paciente deberá mantenerse en una habitación individual, aislada y no podrá salir a otras habitaciones. Las visitas recreativas (familiares y amigos, entre otros) están prohibidas y deberá llevar en todo momento la protección sugerida según recomendación MINSAL u hospital a cargo. El no cumplimiento de estas medidas puede significar la suspensión de visitas presenciales o en su defecto la derivación a hospitalización tradicional según su estabilidad clínica.

- Los instrumentos de medición de signos vitales y evaluación deben ser de un solo uso. Ahora bien, si son compartido entre pacientes, debe ser desinfectado según recomendaciones de IAAS (ANEXO N³). En caso de ser posible, solicitar a los familiares los equipos disponibles en el domicilio para el control de signos vitales.

- En caso de sospecha de sobreinfección bacteriana, dar aviso oportuno al médico de turno (sugerir el inicio del tratamiento adecuado de manera precoz).

- Si no hay respuesta satisfactoria durante los primeros cuatro días de tratamiento se debe solicitar nuevamente visita y evaluación médica para, en conjunto, decidir el reingreso del paciente a hospitalización tradicional, notificando a HD para la coordinación de la llegada del paciente. Se recomienda contar con un kinesiólogo/a coordinador quien deberá gestionar con coordinación de enfermería la llegada del paciente.

- Actualmente, la evidencia ha demostrado que la transmisión de la infección se produce habitualmente por gotitas (producidas al toser, estornudar o hablar) y por contacto de las mucosas (oral, ocular y/o nasal) con material contaminado (5,9,12). Además, puede ocurrir transmisión por aerosol cuando las gotitas respiratorias se mezclan en el aire. Por lo que se 
recomienda la utilización de mascarilla con una eficacia mínima de filtración equivalente a N95 o superior, para la realización de técnicas kinésicas (TTKK) y procedimientos en donde haya riesgo de generación de aerosoles (ANEXO Nº y N5) $(5,12)$.

\section{Consideraciones generales en pediatría}

- Los casos pediátricos alrededor del mundo son, hasta la fecha, escasos. En base a la información disponible, se ha observado que los niños y niñas son diagnosticados de COVID-19 con menor frecuencia y con sintomatología más leve $(13,14)$. Para lo cual se recomienda el seguimiento telefónico evaluando estabilidad clínica.

- En caso de infección no complicada, sólo realizar educación y seguimiento telefónico.

- El cuadro clínico varía desde un cuadro leve y de vías respiratorias altas con uno o más de los siguientes síntomas: disnea, tos o dolor de garganta y/o fiebre hasta un cuadro de neumonía grave con sepsis (ANEXO No6) $(13,15,16)$.

- En caso de complicación o deterioro del cuadro clínico se debe derivar inmediatamente a la urgencia pediátrica previo acuerdo con el personal médico de turno.

\section{Suspensión de visitas}

- Se suspenderán las visitas en los siguientes casos.

- El tutor responsable y/o paciente señala voluntariamente no querer contar con el servicio de HD, debiendo mantener el seguimiento telefónico para control sanitario. En este caso se solicitará firmar acta de rechazo de HD (propia de cada HD).

- Derivación a servicio de urgencias y/o reingreso a hospitalización tradicional.

- Inseguridad del lugar de residencia, para lo cual se recomienda en primera instancia reprogramar la visita.

- En caso de incumplimiento de las indicaciones generales y/o kinésicas de parte del paciente y/o tutores responsables, se recomienda suspensión de visitas total, dando aviso a su coordinación, pero manteniendo el seguimiento telefónico.

\section{Alta kinésica del paciente adulto o pediátrico}

Según acuerdo con equipo médico, existirán 2 tipos de alta:

- Alta con resultado negativo: Paciente con resultado negativo y clínicamente estable y que ingreso a HD por patología respiratoria. Al alta se recomienda continuar con cuarentena preventiva.

- Alta con resultado positivo: Al cumplir con objetivos kinésicos de HD, no presentar sintomatología respiratoria y cumplir criterios de estabilidad clínica, se le indicará al paciente el alta y mantener cuarentena. La toma de una segunda muestra para confirmación de COVID-19 dependerá de los criterios médicos. En caso de persistir con síntomas post alta de HD, se sugiere acudir a servicio de atención de urgencia.

- Para protocolizar y/o estandarizar el alta y toma de muestra de PCR, se recomienda adaptar a su HD el flujograma para paciente "Recuperado" del ANEXO Nº7 (17). 


\section{PROCEDIMIENTOS}

- Lavado clínico de mano: Seguir indicaciones de ANEXO N8. Como segunda opción, o en caso de que las condiciones de higiene en domicilio no sean las adecuadas, se puede utilizar alcohol gel al 70\% frotando por 30 segundos o hasta que el alcohol gel se seque completamente (9).

- EPP: Su colocación y retiro se debe realizar según indicaciones del ANEXO №2. En relación con el uso específico de mascarilla N95 o superior, se recomienda una vez instalada realizar una "prueba de ajuste" (ANEXO N9) para verificar el correcto cierre hermético y que no exista filtración de aire, dar un uso máximo de 8 horas continuas (18). No deberían reutilizarse, sin embargo, en caso de escasez de este elemento se recomienda que sea guardada en una bolsa idealmente de papel o un recipiente plástico con tapa, manipulándola solo por las bandas elásticas, y con un tiempo máximo de 24 horas de uso intermitente. Tener en consideración que el riesgo más significativo de la reutilización de las mascarillas N95, es la transmisión por contacto al tocar la superficie contaminada de la mascarilla. Identificar con nombre del kinesiólogo/a, el tiempo aproximado de uso y la fecha $(19,20)$.

- Oxigenoterapia: La administración de oxígeno se considera un procedimiento generador de aerosoles de bajo riesgo y por lo tanto adecuado para pacientes COVID-19 positivos (6).

- Se recomienda usar una máscara facial, evitando la naricera, con un flujo de oxígeno de hasta $5 \mathrm{~L} / \mathrm{min}$, o una máscara Venturi de hasta el $60 \%$ de $\mathrm{FiO} 2$. Si se utiliza una naricera se sugiere la adición de una mascarilla quirúrgica que cubra nariz y boca del paciente, evitando una mayor dispersión de gotas, la cual debe cambiarse cada $6-8$ horas. $(4,16)$

- Todos los insumos que se requieran para la oxigenoterapia (naricera, mascarilla, humidificador, extensión de naricera, entre otros) deben ser eliminados en la basura según protocolos de su HD e IAAS de su hospital.

- En pacientes con inestabilidad clínica según criterios del ANEXO $N^{\circ} 1$, debe iniciarse oxigenoterapia, ajustando el flujo hasta alcanzar una saturación de oxígeno capilar adecuada a la edad y estado del paciente (6).

- En caso de retirar un concentrador y/o cilindro tipo E de oxígeno de un domicilio, debe ser desinfectado según recomendaciones IAAS del ANEXO N³ y llevado a la UHD privilegiando la posición vertical dentro de lo posible. Entregar al área encargada en su HD, especificando que se encuentra totalmente desinfectado.

- Nebulización: Se recomienda privilegiar siempre el uso de IDM con aerocámara $(6,8)$. En caso de que se decida realizar una nebulización, se debe mantener distanciamiento con el paciente y seguir las siguientes recomendaciones:

- Utilizar una habitación individual, con ventilación natural o si fuera posible y seguro para el paciente realizarla fuera del domicilio (patio). La puerta de la habitación deberá permanecer siempre cerrada.

- Luego de terminar nebulización, y si fue realizada en una habitación, se debe ventilar sin el ingreso de personas por al menos 2 horas y luego solicitar limpieza de las superficies por parte de familiares.

- En caso de realizarla en pacientes conectados a VMNI, se considerará como primera opción los nebulizadores de malla vibrante con adaptación al codo de la interfase, en segunda opción el nebulizador de malla con una pieza en T al circuito del VMNI y como última opción el nebulizador tipo jet, ya que la dispersión de aerosoles es mayor (20). 
- Succión de secreciones: Seguir protocolo establecido por su HD, a lo cual se debe agregar:

- Utilización de EPP por el kinesiólogo y el asistente: Mascarilla N95 o superior, pechera manga larga desechable o traje de seguridad, guantes de procedimiento (estériles de ser necesario), cubre calzado, gorro desechable, escudo facial y antiparras $(5,9)$.

- Se recomienda el uso de circuito de succión cerrado $(6,8)$.

- Desechar todos los insumos utilizados (sonda de aspiración y silicona de succión) según protocolo de su HD.

- En caso de disponibilidad, designar y entregar una bomba de uso exclusivo para el paciente.

- La bomba de aspiración portátil debe limpiarse y desinfectarse según recomendaciones del ANEXON³ y guardada en su contenedor habitual.

- Ventilación mecánica no invasiva (VMNI): Inicialmente no está recomendado el uso de este tipo de soporte ventilatorio en pacientes que presenten una insuficiencia respiratoria aguda severa en domicilio. Frente a este requerimiento, se debe hacer la derivación al servicio de urgencias correspondiente. Sin embargo, en caso de que el paciente sea derivado a HD con VMNI se recomienda seguir las siguientes indicaciones:

- Gestionar visitas médicas y de enfermería para eventual toma de gases arteriales (GSA).

- Utilizar los mismos parámetros con los cuales fue derivado desde el interior del hospital, ajustándolos a la baja.

- En caso de deterioro clínico se recomienda el siguiente manejo inicial $(4,21,22)$ :

- Titular la FiO2 para conseguir una SpO2 entorno al 92\%.

- Utilizar EPAP según evolución clínica y respuesta del paciente, además de presiones de soporte bajas (con el objetivo de obtener un volumen corriente $<9$ $\mathrm{ml} / \mathrm{kg}$ de peso ideal). Evaluar respuesta en máximo una hora, si no es satisfactoria se debe derivar de inmediato al servicio de atención de urgencia.

\section{Recomendaciones específicas durante el uso de VMNI}

Si el paciente es derivado desde hospitalización tradicional y/o conectado en domicilio a VMNI, se recomienda seguir las siguientes precauciones (23):

- Utilizar preferiblemente configuraciones de doble rama, debido a que favorecen que el sistema (circuito respiratorio tanto inspiratorio como espiratorio) sea hermético. Se deberán colocar filtros antimicrobianos de alta eficiencia en la rama espiratoria para evitar la contaminación desde el paciente al ventilador.

- En caso de no disponer de sistemas de doble rama deberemos colocar en el orificio espiratorio un filtro antimicrobiano de alta eficiencia.

- En caso de no poder acoplar un filtro antimicrobiano de alta eficiencia en el orificio espiratorio, se deberá colocar este mismo entre la interfase paciente/ventilador (sin orificios espiratorios) y el circuito.

- Interfases: Se recomiendan sin orificio espiratorio y no utilizar puertos accesorios si los hubiera, además evitar el puerto anti-asfixias con la salvedad de tener una mayor vigilancia del paciente ante posibles fallas del equipo. En orden de preferencia se debe utilizar interfaces tipo helmet, mascara facial total y oro nasa $(21,23)$.

- Se debe monitorizar y/o realizar seguimiento al paciente de forma permanente. 
- Cánula nasal de alto flujo (CNAF): No se recomienda su utilización en domicilio, sin embargo, en caso de derivación desde hospitalización tradicional, se sugiere preferir por sobre VMNI $(12,21,23)$.

- En caso de utilizar, se recomienda un flujo de al menos $50 \mathrm{~L} / \mathrm{min}$ y $\mathrm{FiO} 2$ hasta $60 \%$. La cánula nasal debe estar bien posicionada dentro de las fosas nasales y se debe agregar una mascarilla quirúrgica sobre las cánulas nasales que cubren boca y nariz del paciente. La mascarilla quirúrgica debe cambiarse al menos cada 6 a 8 horas $(4,16)$.

- Se debe monitorizar y/o realizar seguimiento al paciente de forma permanente.

- Manejo de Traqueostomía (TQT): La presencia de una traqueostomía y los procedimientos relacionados con su manejo son potencialmente, generadores de aerosoles (12). Se recomienda seguir protocolo establecido por su HD y además considerar:

- Utilizar todas las medidas de protección para la transmisión del virus por vía aérea.

- Las pruebas de fuga y limpieza/cambios de la cánula interna (endo-cánula) y/o cánula completa deben evitarse, debido a que pueden generar aerosoles, solo realizarlos en caso de ser una urgencia y no de manera rutinaria (12).

- Se recomienda la aspiración con sistemas cerrados de succión.

- El uso de entrenamiento de la musculatura respiratoria y/o válvulas de fonación no deben de realizarse hasta que el paciente haya superado la fase aguda de la infección y el riesgo de transmisión se haya reducido $(6,12)$.

- Utilizar filtros HME (humidificadores pasivos) por 24 horas. En el caso de utilizar oxigenoterapia se conectará a este mismo.

- Toma de muestras biológicas:

- Previo a la toma de muestra se solicitará aseo bucal del paciente.

- Asegurarse que el paciente tiene secreciones y que las puede expectorar de manera independiente. En este caso, el kinesiólogo/a no intervendrá en la tomar de la muestra de esputo (6).

- Si la intervención del Kinesiólogo/a es necesaria para facilitar la extracción de la muestra del esputo, el kinesiólogo/a tendrá que llevar el EPP indicados cuando existe riesgo de transmisión por vía aérea. El manejo de la muestra del esputo debe seguir las políticas de aplicación de su hospital.

- No se recomienda el uso de nebulización con solución hipertónica para la inducción de esputo por su alto riego de dispersión de aerosol.

- Para la toma de muestra específica para COVID-19 se debe seguir protocolo establecido por su HD.

- Para la toma de muestra de baciloscopia (BK) y cultivo de secreciones se recomienda seguir protocolo para toma de muestra de paciente con sospechoso o confirmado para COVID-19de su HD, combinándolo con el de aspiración de secreciones, de ser necesario.

- TTKK (Técnicas Kinésicas): No hay evidencia suficiente que respalde aplicarlas de forma general y/o preventiva en pacientes con COVID-19. De todos modos, se recomienda realizar los procedimientos con criterio clínico específico para cada caso, sopesando el riesgo frente al beneficio de las intervenciones, teniendo siempre en consideración que muchas de las técnicas utilizadas en la kinesiterapia respiratoria, pueden generar aerosoles, con potencial riesgo de transmisión del virus por vía área debiendo tener las precauciones ya expuestas (6).En el caso de pacientes con antecedentes oncológicos, se contraindican las técnicas kinésicas que impliquen compresión de la parrilla costal o que involucren golpes en el tórax. 
- Tos: El pacientes y kinesiólogo/a deben aplicar las medidas necesarias para evitar la dispersión de aerosoles durante la tos, tratando de evitar estas técnicas si el paciente puede toser de manera independiente $(4,6,8)$.

- Medidas de higiene respiratoria: Usar mascarilla en caso de disponer, cubrir la boca con antebrazo, girar la cabeza, utilizar pañuelo desechable (6).

- Privilegiar las técnicas de tos que puedan ser mediante asistencia verbal, tratando de mantener una distancia mayor o igual dos metros del paciente dentro de lo posible (6).

- Dependiendo de las condiciones del paciente y con el fin de evitar el contacto cercano, se recomienda este orden de prioridad en la aplicación de técnicas de tos: tos dirigida, tos asistida y tos provocada.

- Técnicas de ventilación:

- Ejercicio con debito inspiratorio controlado (EDIC), no existe evidencia de generación de aerosol.

- Se debe evitar la hiperinsuflación manual y técnicas asociadas. Sin embargo, se recomienda el uso de filtros si se decide su realización (21).

- Cambios de posición con un enfoque de ventilación en distintas zonas pulmonares, no tiene evidencia de generación de aerosol.

- Husmeos, respiración diafragmática y seseos, no existe evidencia de riesgo de aerosol.

- Técnicas de permeabilización: La mayoría de estas técnicas están asociadas a generación de tos y/o a su estimulación, por lo que se recomienda precaución ante la posibilidad de generación de aerosol. Sólo aplicarlas en caso de evidencia de retención de secreciones con dificultad para expectorar y/o Ineficacia de la tos (8).

- Técnicas de espiración forzada (TEF).

- Aceleración del flujo espiratorio (AFE).

- Espiración lenta con glotis abierta infra lateral (ETGOL).

- Ciclos activos respiratorios (CAR).

- Espiración lenta prolongada (ELPR).

- Drenaje autógeno (DA).

- Desobstrucción rinofaríngea retrograda (DRR).

- Presión/Descompresión.

- Técnicas instrumentales $(6,8)$ :

- Cuando se requieran dispositivos para realizar el tratamiento, siempre que sea posible, utilizar dispositivos de un solo uso y desechables. Los dispositivos reutilizables deben evitarse siempre que sea posible.

- Los dispositivos que generan presión positiva al fin de la espiración con o sin oscilación podrían generar aerosol.

- No existe evidencia sobre el uso de incentivadores inspiratorios en este tipo de pacientes. 
- Evitar el uso de (insuflación/exsuflación mecánica) tipo coughassist, sin embargo, si clínicamente es beneficioso se debe asegurar que los dispositivos pueden ser desinfectados después de su uso y/o proteger los dispositivos con filtros antibacterianos.

- Cambiar circuitos en caso de tener insumos disponibles.

- Rehabilitación cardio respiratoria (24): Se recomienda en todos los pacientes dados de alta por COVID-19 desde la hospitalización tradicional. Realizar rehabilitación motora y respiratoria, sobre todo en aquellos pacientes con antecedentes de hospitalización prolongada en unidades de paciente crítico.

- Pacientes ingresados a HD posterior a un cuadro leve: La rehabilitación se basa principalmente en la recuperación sus cualidades físicas y psicológicas. Se recomienda indicar el ejercicio aeróbico para recuperar gradualmente su capacidad de realizar actividades de la vida diaria y participación.

- Pacientes ingresados a HD luego de un cuadro grave/crítico: De acuerdo con la evidencia actual, los pacientes con COVID-19 pueden tener una condición física deficiente que se traduce en disnea después de una actividad y atrofia muscular (incluidos los músculos respiratorios y del tronco), como también de otros problemas funcionales y trastornos psicológicos como el síndrome de estrés postraumático. Por lo que deben recibir tratamiento de rehabilitación respiratoria y musculoesquelética.

En conjunto con el equipo médico de HD se debe decidir el riesgo/beneficio del inicio de la rehabilitación respiratoria en los pacientes con hipertensión pulmonar, miocarditis, insuficiencia cardíaca congestiva, trombosis venosa profunda, fracturas inestables y otras enfermedades. Se recomiendan los siguientes criterios de exclusión y detención del ejercicio:

- Criterios de exclusión:

- Frecuencia cardíaca de reposo >100 latidos/min

- Presión arterial menor a 90/60 mmHg o mayor a 140/90 mmHg.

- Saturación de oxígeno $\leq 95 \%$. Evaluar en cada caso según la saturación de oxígeno basal del paciente.

- Criterios de finalización del ejercicio:

- Temperatura corporal $>37,2^{\circ} \mathrm{C}$.

- Aumento de los síntomas respiratorios, fatiga y falta de alivio después del descanso.

- Opresión y/o dolor en el pecho, tos severa, mareos, dolor de cabeza, visión borrosa, palpitaciones y/o sudoración. 


\section{ANEXOS}

\section{Anexo n. 1: Criterios de estabilidad y niveles de gravedad de las infecciones respiratorias (12)}

Criterios de estabilidad clínica

\begin{tabular}{|l|l|}
\hline \multicolumn{1}{|c|}{ Constante } & \multicolumn{1}{c|}{ Valores de estabilidad } \\
\hline Frecuencia cardiaca & $<100 \mathrm{Ipm}$ \\
\hline Frecuencia respiratoria & $<24 \mathrm{RPM}$ \\
\hline Temperatura axilar & $<37,29 \mathrm{C}$ \\
\hline Presión arterial sistólica & $\$ 90 \mathrm{mmHg}$ \\
\hline $\mathrm{SaO}_{2}$ & $\$ 90 \%$ si no habia insuficiencia respiratoria previa \\
\hline Nivel de conciencia & Adecuado \\
\hline
\end{tabular}

Niveles de gravedad de las infecciones respiratorias y sus definiciones (El recuadro rojo señala es el nivel de gravedad que se puede atender en domicilio, el resto debe ser derivado al servicio de urgencia con prontitud).

\begin{tabular}{|c|c|}
\hline Nivel de gravedad & Descripción \\
\hline $\begin{array}{l}\text { Enfermedad no } \\
\text { complicada }\end{array}$ & $\begin{array}{l}\text { Cursa con sintomas locales en vias respiratorias altas y puede cursar con síntomas } \\
\text { inespecificos como fiebre, dolor muscular o sintomas atipicos en ancianos }\end{array}$ \\
\hline Neumonía leve & $\begin{array}{l}\text { Confirmada con radiografía de tórax y sin signos de gravedad. SaO } \text { aire ambiente } \\
>90 \% \text {. CURB } 65 \leq 1\end{array}$ \\
\hline Neumonia grave & $\begin{array}{l}\text { Fallo de } \geq 1 \text { organo o } \mathrm{SaO}_{2} \text { aire ambiente }{ }^{90 \%} \% \\
\text { of frecuencia respiratoria de } \geq 30\end{array}$ \\
\hline Distrés respiratorio & $\begin{array}{l}\text { Hallazgos clínicos, radiográficos infiltrados bilaterales + déficit de oxigenación: } \\
\text {-Leve: } 200 \mathrm{mmHg}<\mathrm{PaO}_{2} / \mathrm{FiO}_{2} \leq 300 \\
\text {-Moderado: } 100 \mathrm{mmHg}_{2}<\mathrm{PaO}_{2} / \mathrm{FiO}_{2} \leq 200 \\
\text {-Grave: } \mathrm{PaO}_{2} / \mathrm{FiO}_{2} \leq 100 \mathrm{mmHg} \\
\mathrm{Si}_{\mathrm{PaO}} \text { no disponible } \mathrm{SaO}_{2} / \mathrm{FiO}_{2} \leq 315\end{array}$ \\
\hline Sepsis & $\begin{array}{l}\text { Definida como disfunción orgánica y que puede ser identificada como un cambio agudo } \\
\text { en la escala SOFA }>2 \text { puntos. } \\
\text { Un quick SOFA ( } Q S O F A) \text { con } 2 \text { de las siguientes } 3 \text { variables clinicas puede identificar a } \\
\text { pacientes graves: Glasgow } 13 \text { o inferior, Presión sistólica de } 100 \mathrm{mmHg} \text { o inferior y } \\
\text { frecuencia respiratoria de } 22 / \text { min o superior. } \\
\text { La insuficiencia orgánica puede manifestarse con las siguientes alteraciones: } \\
\text {-Estado confusional agudo } \\
\text {-Insuficiencia respiratoria } \\
\text {-Reducción en el volumen de diuresis } \\
\text {-Taquicardia } \\
\text {-Coagulopatía } \\
\text {-Acidosis metabólica }\end{array}$ \\
\hline
\end{tabular}

\section{Anexo n. ${ }^{\circ}$ 2: Colocación y Retiro de EPP (18)}

Secuencias de colocación de EPP durante atención de casos sospechosos o confirmados 


\begin{tabular}{|c|c|}
\hline Paso 1 & Retirar joyas relojes y otros elementos personales, incluidos los teléfonos celulares \\
\hline Paso 2 & Realizar lavado clínico de manos \\
\hline Paso 3 & $\begin{array}{l}\text { Colocar la bata/delantal impermeable } \\
\text { Cubrir ambos brazos por completo e introduzca el dedo pulgar en la cinta ubicada en el } \\
\text { extremo distal de las mangas (gancho), si es que el modelo lo tiene, de modo de fijar la manga } \\
\text { a la mano. Anudar los lazos firmemente en la región posterior a la altura de la cintura, nunca en } \\
\text { la región anterior. }\end{array}$ \\
\hline Paso 4 & Colocar el respirador o mascarilla tipo N95, FFP2 o equivalente. \\
\hline Paso 5 & $\begin{array}{l}\text { Realizar prueba de control de sellado con la mascarilla o respirador tipo N95, FFP2 o } \\
\text { equivalente. }\end{array}$ \\
\hline Paso 6 & $\begin{array}{l}\text { Colocar la protección ocular: antiparras o escudo facial } \\
\text { Colocar las antiparras sobre los ojos asegurando que no deje espacios abiertos en los bordes } \\
\text { entre la piel de la cara y las antiparras mismas. Pasar la fijación de (cinta, elástico u otra) hacia } \\
\text { la región occipital. } \\
\text { Hay que asegurar que se produzca un ajuste cómodo. Si las antiparras se fijan a los lentes } \\
\text { ópticos y éstos se salen al momento de sacarse las antiparras, debe preferirse el uso de } \\
\text { escudos faciales. } \\
\text { Colocar el escudo facial frente a la cara asegurando que no queden espacios abiertos entre la } \\
\text { fijación a nivel de la frente. Utilizar el mecanismo de fijación de modo que quede firme y no se } \\
\text { desplace, pero sin apretar demasiado para que se produzca un ajuste cómodo. }\end{array}$ \\
\hline Paso 7 & $\begin{array}{l}\text { Colocar los guantes } \\
\text { Guante de procedimiento y/o estéril según corresponda, hasta los antebrazos para obtener el } \\
\text { ajuste y que no se desplacen o se salgan. }\end{array}$ \\
\hline Paso 8 & $\begin{array}{l}\text { Colocar la pechera impermeable y desechable, anudada a la cintura (sólo si se prevé } \\
\text { exposición a gran volumen de fluidos). }\end{array}$ \\
\hline
\end{tabular}

Secuencias de retiro de EPP

\begin{tabular}{|l|l|}
\hline Paso 1 & $\begin{array}{l}\text { Retiro de pechera y guantes. } \\
\text { Sin retirar los guantes, con ambas manos, desde la cintura y con movimientos firmes tracciones } \\
\text { hacia adelante para desprender los lazos. } \\
\text { Repita instrucción anterior para desprender amarras de cuello. } \\
\text { Tomar el delantal alejado del cuerpo a la máxima distancia que le permita sus brazos, por su } \\
\text { cara externa enrollándolo de manera circular, siempre mantener enfrentando a la cara interna } \\
\text { (que estaba en contacto con el cuerpo). Al llegar a las manos, retire guantes: desprenda desde } \\
\text { la muñeca, para el retiro del primer guante. } \\
\text { El segundo guante se retira introduciendo un dedo por dentro del guante, contactando solo } \\
\text { superficie interior del mismo. } \\
\text { Desechar en el contenedor de residuos }\end{array}$ \\
\hline
\end{tabular}




\begin{tabular}{|l|l|}
\hline Paso 2 & $\begin{array}{l}\text { Primer lavado d de manos } \\
\text { Fricción con alcohol gel al menos } 20 \text { segundos o lavado clínico de manos, al menos } 40 \\
\text { segundos de duración. }\end{array}$ \\
\hline Paso 3 & $\begin{array}{l}\text { Retiro Escudo facial o/ y Antiparras } \\
\text { Con una mano, tomar la fijación del escudo o antiparras en la región posterior de la cabeza y } \\
\text { traccionar, desplazar la fijación por sobre la cabeza, con tensión suficiente para evitar tocar } \\
\text { la cara y la mascarilla hasta sacarla. Durante el retiro de las antiparras mantenga sus ojos } \\
\text { cerrados. } \\
\text { Dejar EPP en la caja destinada a limpieza de insumos }\end{array}$ \\
\hline Paso 4 & $\begin{array}{l}\text { Retiro de mascarilla quirúrgica o N95 } \\
\text { Corte las amarras de la mascarilla quirúrgica desde la zona posterior y retire siempre hacia } \\
\text { delante, sin soltar amarras elimine en contenedor de desecho. } \\
\text { Si es mascarilla con elástico, retire de atrás de pabellón auricular y siempre hacia delante, sin } \\
\text { soltar amarras, elimine en contenedor de desecho }\end{array}$ \\
\hline Paso 5 & $\begin{array}{l}\text { Segundo lavado de manos } \\
\text { Fricción con alcohol gel al menos 20 segundos o lavado clínico de manos, al menos } 40 \\
\text { segundos de duración. }\end{array}$ \\
\hline
\end{tabular}

\section{Anexo n. ${ }^{\circ}$ 3: Recomendación IAAS para limpieza de equipos}

Todos los puntos deben ser realizados con paños desechables, en el domicilio del paciente y/o en su unidad de HD en el lugar destinado a esto.

13. Lavar con un paño con solución jabonosa (jabón, integrasa etc..), con el objetivo de retirar toda la materia orgánica. Teniendo especial cuidado con los equipos electrónicos.

14.Con un paño humedecido en agua, retirar toda la solución jabonosa (no aplicar agua directamente) en orden y teniendo precaución de seguir solo una dirección.

15. Aplicar cloro con un atomizador al 10\% en una proporción de $950 \mathrm{ml}$ de agua y $50 \mathrm{ml}$ de cloro o $1 / 2$ litro de agua con $4 \mathrm{~g}$ de cloro.

16. Los siguientes equipos deben seguir la secuencia anterior:

- Concentrador y/o cilindro de transporte de oxígeno.

- Bomba de aspiración de secreciones incluyendo su receptáculo.

- Protector facial.

- Ventiladores Mecánicos (sin incluir interface ni corrugado).

- CNAF, sin incluir insumos desechables.

- Nebulizador portátil.

Los elementos de evaluación como fonendoscopio, saturómetro, tensiómetro y termómetro deben ser limpiados con alcohol al 70\% y luego desinfectados con la misma solución teniendo precaución de seguir una sola dirección.

\section{Anexo n. ${ }^{\circ}$ 4: Procedimientos con alto y bajo riesgo de aerosol (12)}

En los recuadros en rojo se destacan los procedimientos más frecuentemente realizados en hospitalización domiciliaria. 


\begin{tabular}{|c|c|}
\hline $\begin{array}{l}\text { Procedimientos de BAJO RIESGO de } \\
\text { transmisión vírica }\end{array}$ & $\begin{array}{l}\text { Procedimientos de ALTO RIESGO de } \\
\text { transmisión vírica }\end{array}$ \\
\hline $\begin{array}{l}\text { - Colocación de tubo de Guedel } \\
\text { - Colocación de mascarilla facial de } \\
\text { oxigenoterapia con filtro espiratorio } \\
\text { - Compresión torácica }\end{array}$ & $\begin{array}{l}\text { Aerosolterapia, nebulizacion } \\
\text { - Gafas nasales de alto flujo } \\
\text { Ventilación manual con mascarilla } \\
\text { Ventilación no invasiva CPAP/BiPAP }\end{array}$ \\
\hline $\begin{array}{l}\text { de marcapasos transcutáneo } \\
\text { - Inserción de vía venosa o arterial }\end{array}$ & $\begin{array}{l}\text { Intubación orotraqueal } \\
\text { - } \quad \text { Traqueotomía quirúrgica }>\text { percutánea } \\
\text { - Broncoscopia, gastroscopia }\end{array}$ \\
\hline $\begin{array}{l}\text { Administración de fármacos o fluidos } \\
\text { intravenosos }\end{array}$ & $\begin{array}{l}\text { - Aspiración de secreciones } \\
\text { - Resucitación cardiopulmonar }\end{array}$ \\
\hline
\end{tabular}

\section{Anexo n. ${ }^{\circ}$ : Procedimientos susceptibles a generar aerosoles y estrategias sugeridas para disminuir el riesgo (12)}

En los recuadros en rojo se destacan los procedimientos más frecuentemente realizados en hospitalización domiciliaria.

\begin{tabular}{|c|c|}
\hline Procedimiento & Estrategia \\
\hline Aspiración de secreciones respiratorias & $\begin{array}{l}\text { Limitar a las imprescindibles } \\
\text { Aspiración cerrada si VM }\end{array}$ \\
\hline Aerosolterapia & Evitar si es posible \\
\hline Toma de muestras respiratorias & Limitar a las imprescindibles \\
\hline Lavado broncoalveolar & Evitar si es posible \\
\hline Oxigenoterapia de alto flujo & Evitar \\
\hline Ventilación no invasiva (VNI) & $\begin{array}{l}\text { - Evitar si es posible. } \\
\text { - En caso necesario asegurar el sellado } \\
\text { adecuado de la interfase } \\
\text { - Uso de VNI con doble tubuladura y filtros de } \\
\text { alta eficacia. }\end{array}$ \\
\hline $\begin{array}{l}\text { Ventilación manual con mascarilla y bolsa } \\
\text { autoinflable }\end{array}$ & $\begin{array}{l}\text { Evitar la ventilación con mascarilla y bolsa } \\
\text { autoinflable. En caso necesario, utilizar filtro de } \\
\text { alta eficiencia que impida la contaminación vírica, } \\
\text { entre la bolsa autoinflable y la mascarilla, sellar } \\
\text { bien la mascarilla para evitar fugas y utilizar } \\
\text { pequeños volúmenes corrientes. } \\
\text { Descontaminar todo el material utilizado después del } \\
\text { uso seeún los protocolos del centro. }\end{array}$ \\
\hline Intubación & $\begin{array}{l}\text { - Si es necesario se preoxigenará con oxigeno al } \\
100 \% \text { a través de mascarilla facial. } \\
\text { - Se realizará con una secuencia rápida de } \\
\text { intubación y por personal experto para } \\
\text { minimizar el tiempo y el número de intentos } \\
\text { del procedimiento de intubación. } \\
\text { - Anticipar en la medida de lo posible } \\
\text { - Uso de tubos con aspiración subglótica }\end{array}$ \\
\hline Ventilación mecánica (VM) & $\begin{array}{l}\text { - Se pondrán los filtros de alta eficiencia que } \\
\text { impidan la contaminación vírica tanto en la } \\
\text { rama inspiratoria como en la espiratoria. } \\
\text { - Se usará el sistema de aspiración cerrada de } \\
\text { secreciones } \\
\text { - Uso de intercambiador de calor y humedad } \\
\text { con filtro de alta eficacia que impida la }\end{array}$ \\
\hline
\end{tabular}




\section{Anexo n. 6: Síndromes clínicos asociados a infección respiratoria viral aguda en paciente pediátricos (12)}

El recuadro rojo señala el nivel de gravedad que se puede atender en domicilio, el resto debe ser derivado al servicio de urgencia con prontitud.

\begin{tabular}{|c|c|}
\hline $\begin{array}{l}\text { Infección no } \\
\text { complicada }\end{array}$ & $\begin{array}{l}\text { - Los pacientes con infección viral no complicada del tracto respiratorio superior pueden presentar } \\
\text { síntomas inespecíficos, como fiebre, tos, dolor de garganta, congestión nasal, malestar general, } \\
\text { dolor de cabeza, dolor muscular o malestar general. } \\
\text { - No existen signos de deshidratación, sepsis o dificultad respiratoria. }\end{array}$ \\
\hline $\begin{array}{l}\text { Infección leve de } \\
\text { vias bajas }{ }^{2}\end{array}$ & $\begin{array}{l}\text { - Tos, dificultad respiratoria con taquipnea, pero sin signos de gravedad clinica o neumonia severa. } \\
\text { - Criterios de taquipnea (en respiraciones / min): } \\
<2 \text { meses } 260 \text { RPM } \\
2-11 \text { meses, } 250 \text { RPM } \\
1-5 \text { años, } \geq 40 \text { RPM } \\
\text { Adultos/adolescentes > } 30 \text { RPM } \\
\text { - Saturación ambiental >92\%. Pueden o no tener fiebre }\end{array}$ \\
\hline $\begin{array}{l}\text { Infección grave de } \\
\text { vías bajas }^{2}\end{array}$ & $\begin{array}{l}\text { Tos o dificultad respiratoria y al menos uno de los siguientes: } \\
\text { - Cianosis central o } \mathrm{SaO}_{2}<92 \% \text { ( }<90 \% \text { en prematuros } \\
\text { - Dificultad respiratoria severa: quejido, aleteo nasal, tiraje supraesternal, retracción torácica severa } \\
\text { o disociación toraco-abdominal. } \\
\text { - Produce incapacidad o dificultad para alimentación. } \\
\text { - Produce disminución del estado de conciencia, letargo o pérdida de conocimiento o convulsiones. } \\
\text { - Taquipnea severa (en respiraciones/min): } 270 \mathrm{rpm} \text { en menores de } 1 \text { año; } 250 \text { rpm en mayores de } 1 \\
\text { año. } \\
\text { - Gasometria arterial: } \mathrm{PaO}_{2}<60 \mathrm{mmHg}, \mathrm{PaCO}_{2}>50 \mathrm{mmHg} \text {. } \\
\text { El diagnóstico es clínico; las imágenes de radiografia de tórax pueden excluir complicaciones } \\
\text { (atelectasias, infilltrados, derrame). }\end{array}$ \\
\hline $\begin{array}{l}\text { Otras } \\
\text { manifestaciones } \\
\text { asociadas a } \\
\text { cuadros graves }\end{array}$ & $\begin{array}{l}\text { - Trastornos de la coagulación (tiempo prolongado de protrombina y elevación de dímero-D), daño } \\
\text { miocárdico (aumento de enzimas miocárdica, camblos de ST-T en el electrocardiograma, } \\
\text { cardiomegalia e insuficiencia cardiaca), insuficiencia renal, disfunción gastrointestinal, elevación } \\
\text { de enzimas hepática y rabdomiolisis. }\end{array}$ \\
\hline
\end{tabular}

\section{Anexo n. 7: Flujograma de toma de muestra PCR-RT para consideración de paciente como "Recuperado" (17)}

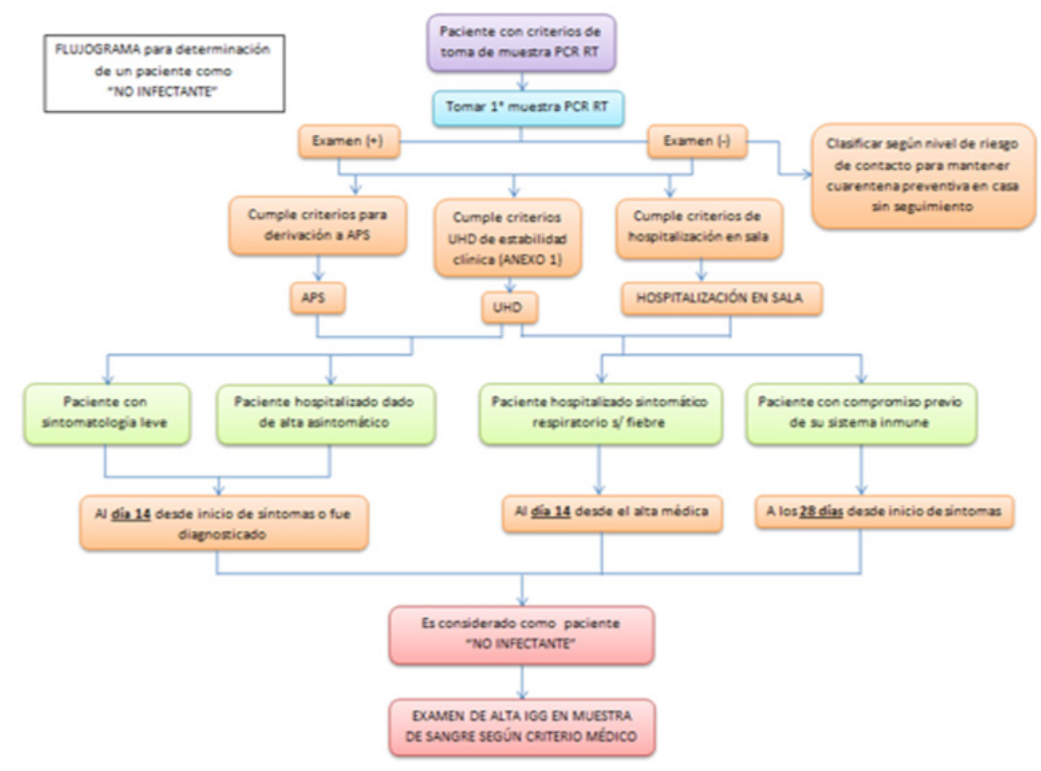

AP: Atención Primaria de salud

UHD: Unidad de Hospitalización Domiciliaria 
Anexo n. ${ }^{\circ}$ 8: Lavado clínico de manos (18)

\section{0}

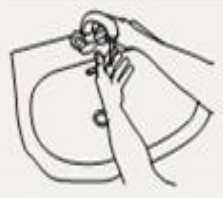

Mojese las manos.

3

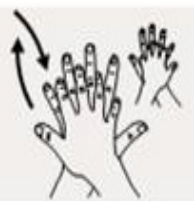

Froblese la paima de la mano derecha contra el dorso de la mano izquierda entrelazando los dedos, y viceversa.

\section{6}

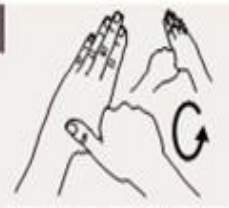

Rodeando el pulgar izquierdo con la paina de la mano detecha, tró leselo con un moviniento de to tacion, y viceversa.

\section{9}

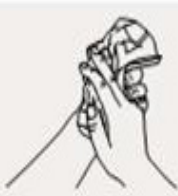

Sequeseiss con uns bslo de un soio uso.
1

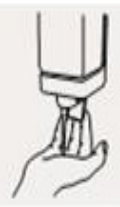

Aplique sufidente jabón para cubrit lodas las superficies de las manos.
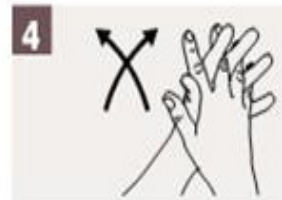

Frólese las paimas de las manos entre si, con los dedos entrelazados.

\section{7}

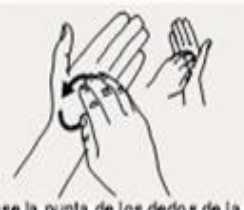

Fiolese la punta de los dedos de la mano derecha contra la paina de la mano izqulerda, haciendo un movimiento de rotación, y viceversa.

\section{0}

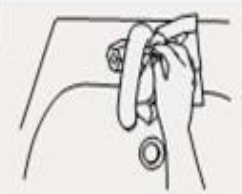

Uilice is ballo para certar el grila.

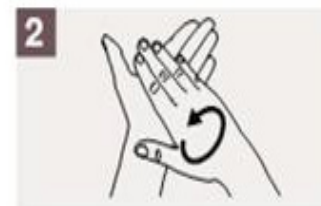

Frólese las paimas de las manos entre sil.

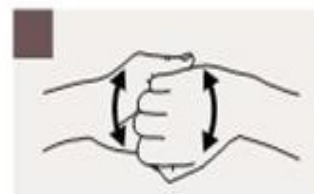

Frólese el dor so de los dedos de uns mano contra la paima de la mano opuesta, manteniendo unidos los

\section{8} dedos.

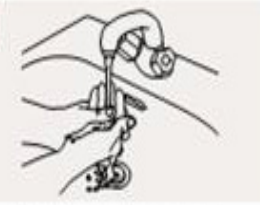

Enjuaguese las manos.

11

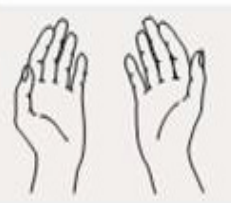

Sus manos sen seguras. 


\section{Anexo n. 9: Prueba de ajuste mascarilla N95 (18)}

\begin{tabular}{|l|l|}
\hline PASO 1 \\
$\begin{array}{l}\text { Sostenga el respirador en la palma de la mano con la parte que cubre la } \\
\text { nariz en la punta de sus dedos permitiendo que las bandas para la } \\
\text { cabeza cuelguen libremente debajo de su mano. }\end{array}$ \\
\hline $\begin{array}{l}\text { PASO } 2 \\
\text { Coloque el respirador debajo de su mentón con la pieza nasal hacia } \\
\text { arriba. }\end{array}$ \\
\hline $\begin{array}{l}\text { PASO } 3 \\
\text { postire la banda superior sobre su cabeza dejándola alta en la parte } \\
\text { colóquela alrededor del cuello por debajo de las orejas }\end{array}$ \\
\hline
\end{tabular}

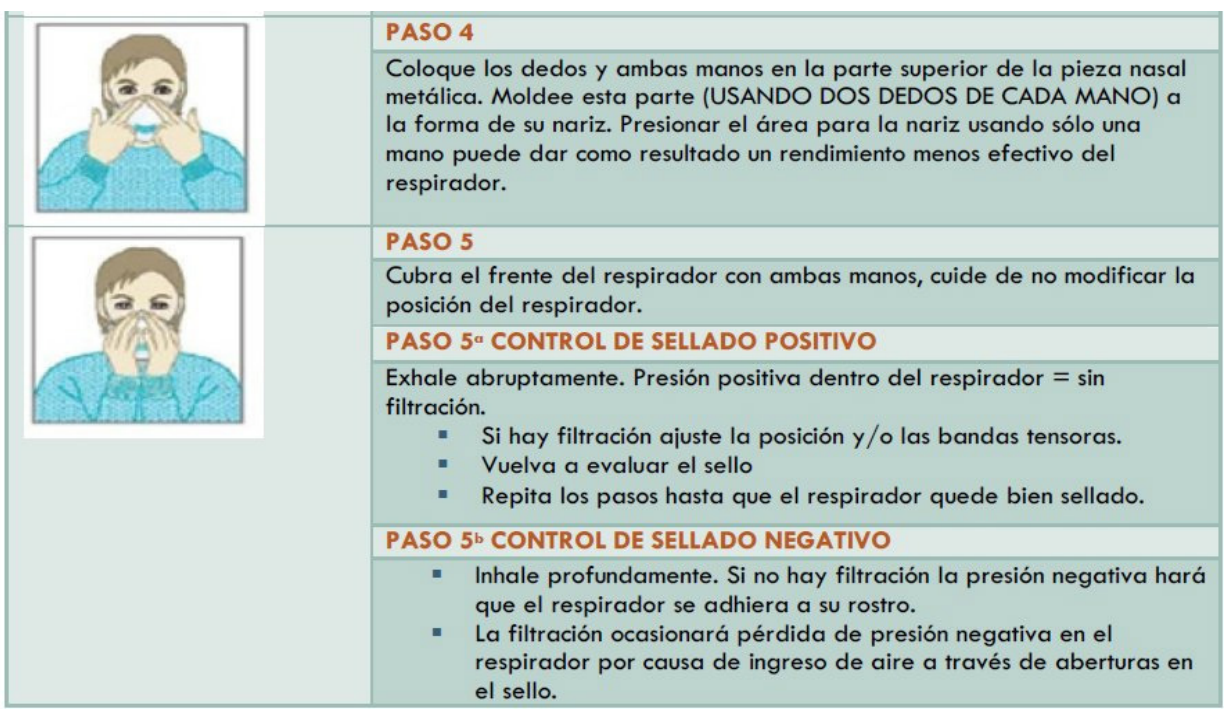




\section{BIBLIOGRAFÍA}

1. Zhou W (editor). The Coronavirus Prevention Handbook: 101 Science based tips that could save your life. New York, United States: Skyhorse Publishing; 2020.

2. Michael J. Loeffelholz, Yi-Wei Tang. Laboratory diagnosis of emerging human coronavirus infections - the state of the art. Emerg Microbes Infect. 2020;9(1):747-56. DOI: 10.1080/22221751.2020.1745095; PMID: 32196430

3. Mujal A, Regalado J, Ruiz M, Mirón M, Basterretxea A, Estrada O, et al. Derivación a hospitalización a domicilio de pacientes con infección por COVID-19. Hosp Domic. 2020;4(2):9-17. DOI: 10.22585/hospdomic.v4i2.104

4. Lazzeri M, Lanza A, Bellini R, Bellofiore A, Cacchetto S, Colombo A, et al. Respiratory physiotherapy in patients with COVID-19 infection in acute setting: a position paper of the Italian association of respiratory physiotherapist (ARIR). Monaldi Arch Chest Dis. 2020;90(1). DOI: 10.4081/ monaldi.2020.128; PMID: 32236089

5. World Health Organization (WHO). Clinical management of severe acute respiratory infection (SARI) when COVID-19 disease is suspected: Interim guidance [monograph on the Internet]. Geneva, Switzerland: WHO; 2020 [consulted 20 may 2020]. WHO REFERENCE NUMBER: WHO/2019-nCoV/clinical/2020.4. Available from: https://bit.ly/2zmgGNN

6. Thomas P, Baldwin C, Bissett B, Boden I, Gosselink R, Granger CL, et al. Physiotherapy management for COVID-19 in the acute hospital setting: clinical practice recommendations. J Physiother. 2020;66(2):73-82. DOI: 10.1016/j.jphys.2020.03.011; PMID: 32312646

7. Freire Figueroa F, Marín Navarro V. Descripción e impacto económico de los pacientes con patologías respiratorias ingresados con indicación de kinesiología en la Unidad de Hospitalización Domiciliaria del Hospital Dr. Sótero del Río. Hosp Domic. 2019;3(4):243-54. DOI: 10.22585/ hospdomic.v3i4.86

8. Arbillaga A, Pardàs M, Escudero R, Rodríguez R, Alcaraz V, Llanes S, et al. Fisioterapia respiratoria en el manejo de paciente con Covid-19: Recomendaciones generales [monografía en Internet]. Barcelona, España: Sociedad Española de Neumología y Cirugía Torácica (SEPAR); 2020 [consultada 20 de mayo 2020]. Disponible en: https://bit.ly/36xBz57

9. Subsecretaría de redes asistenciales (SRA), división de gestión de la red asistencial, departamento de la calidad y seguridad de la atención. Protocolo de referencia para correcto uso de equipos de protección personal en pacientes sospechosos o confirmados de COVID-2019 [monografía en Internet]. 2020.Santiago, Chile: SRA; 2020. Circular C37 N. ${ }^{\circ}$ 1. Disponible en: https://bit.ly/2zXT16n

10. Conti EJ, Monteiro SG. Encuesta sobre kinesiología respiratoria: situación actual en Argentina. Rev Am Med Resp.2009;9:14-20.

11. He M, Yu S, Wang L, Lv H, Qiu Z. Efficiency and Safety of Pulmonary Rehabilitation in Acute Exacerbation of Chronic Obstructive Pulmonary Disease. Med Sci Monit, 2015;21:806-12. DOI: 10.12659/MSM.892769; PMID: 25783889

12. Centro de coordinación de alerta y emergencias sanitarias, Dirección general de salud pública, calidad e Innovación. Manejo clínico del Covid-19: atención hospitalaria [monografía en Internet]. Madrid, España: Ministerio de Sanidad; 2020 [consultada 20 de mayo 2020]. Disponible en: https://bit.ly/36qwV8H 
13. Zhu L, Lu X, Chen L. Possible causes for decreased susceptibility of Children to coronavirus. Pediatric Research; 2020 [Online ahead of print]. DOI:10.1038/s41390-020-0892-8; PMID: 32268343

14. Shereen M, Khan S, Kazmi A, Bashir N, Siddique R. COVID-19 infection: Origin, transmission and characteristics of human coronaviruses. J Adv Res. 2020;24:91-8. DOI: 10.1016/j. jare.2020.03.005

15. Ong J, Tosoni A, Kim Y, Kisoon N, Murthy S. Coronavirus disease in critically ill children: A narrative review of the literature. Pediatric Critical Care Medicine.2020. DOI: 10.1097/ PCC. 0000000000002376 ; PMID: 32265372

16. Calvo C, García López-Hortelano M, de Carlos Vicente JC, Vázquez Martínez JL; Grupo de trabajo de la Asociación Española de Pediatría para el brote de infección por Coronavirus. An Pediatr (Barc). 2020;92(4):241.e1-241.e11. DOI: 10.1016/j.anpedi.2020.02.001; PMID: 32173188

17. Criterios para definir casos recuperados en casos confirmados para Covid-19. Servicio de Salud Coquimbo [monografía en Internet]. Región de Coquimbo, Chile: Consejo Asesor COVID-19; 2020 [consultada 20 de mayo de 2020]; Ord.1, Código: A/00673. Disponible en: https://bit. ly/3gcYA1t

18. Organización Panamericana de la Salud, Organización Mundial de la Salud (OPS/OMS). Prevención y control de infecciones asociadas a la atención de salud: Recomendaciones básicas [monografía en Internet]. Washington, EE. UU.: OPS/OMS; 2017 [consultada 20 de mayo de 2020]; Clasificación NML: WA110. Disponible en: https://bit.ly/3gdBGqN

19. Centers for Disease Control and Prevention (CDC). Recommended guidance for extended use and limited reuse of N95 filtering facepiece respirators in healthcare settings [monograph on the Internet]. Atlanta, USA: National Institute for Occupational Safety and Health; 2020 [consulted 20 may 2020]. Available from: https://bit.ly/3gd0Dmb

20. Cinesi Gómez C, Peñuelas Rodríguez O, Luján M, Egea Santaolalla C, Massa Jiménez F. Recomendaciones de consenso respecto al soporte respiratorio no invasivo en el paciente adulto con insuficiencia respiratoria aguda secundaria a infección por SARSCoV-2 [monografía en Internet]. Madrid, España: Sociedad Española de Anestesiología, Reanimación y Terapéutica del Dolor; 2020 [consultada 20 de mayo 2020]. Disponible en: https://bit.ly/3edOIKq

21. Ferrando C, Mellado R. PNT Soporte respiratorio en pacientes COVID 19 [monografía en Internet]. Barcelona, España: Hospital Clínic Universitari: 2020. [consultada 20 de mayo 2020]. Disponible en: https://bit.ly/3eaeVIC

22. Morand A, Fabre A, Minodier P, Boutin A, Vanel N, Bosdure E, et al. COVID-19 virus and children: What do we know? Arch Pediatr. 2020;27(3):117-8. DOI: 10.1016/j.arcped.2020.03.001;

23. Phua J, Weng L, Ling L, Egi M, Lim C, Divatia JV, et al. Intensive care management of coronavirus disease 2019 (COVID-19): challenges and recommendations. Lancet Respir Med. 2020;8(5):506-17. DOI: 10.1016/S2213-2600(20)30161-2; PMID: 32272080

24. Liu K, Zhang W, Yang Y, Zhang J, Li Y, Chend Y. Respiratory Rehabilitation in elderly patients with COVID-19: A randomized trial controlled study. Complement Ther Clin Pract. 2020;39:101166. DOI: 10.1016/j.ctcp.2020.101166; PMID: 32379637 